\title{
Application of objective clinical human reliability analysis (OCHRA) in assessment of technical performance in laparoscopic rectal cancer surgery: common mistakes and methodological issues
}

\author{
N. K. Francis ${ }^{1}$ J. D. Foster ${ }^{1}$ • E. Salib ${ }^{2}$
}

Received: 23 April 2017/Accepted: 27 April 2017/Published online: 13 May 2017

(C) Springer International Publishing AG 2017

\section{Dear Sir,}

We would like to thank Dr. Sabour for his objective observation about our paper entitled "Application of objective clinical human reliability analysis (OCHRA) in assessment of technical performance in laparoscopic rectal cancer surgery" $[1,2]$. We read his comments with interest.

Our study used a standard methodology, applying an error identification system to a series of recorded laparoscopic rectal surgery procedures and comparing the frequency of identified technical errors with the pathologist's score of the quality of the resection specimen. The choice of statistical methods in our paper was based on professional statistical input which applied a similar approach as in several similar published studies, and is an approach which we believe to be valid. However, as we are currently in the process of finalising a follow-up study on the topic, we will consider applying Sabour's proposed statistical approach in a comparative appraisal of our new findings.

We are most grateful to Dr. Sabour for his interest our study.

\section{Compliance with ethical standards}

Conflict of interest The authors declare that they have no conflict of interest.

Ethical approval Ethical approval is not required for this paper.

Informed consent For this type of study formal consent is not required.

\footnotetext{
J. D. Foster

jakedeclanfoster@hotmail.com

Department of Surgery, Yeovil District Hospital, Yeovil, UK

2 Faculty of Health and Life Sciences, University of Liverpool, Brownlow Hill, Liverpool, UK
}

\section{References}

1. Foster JD, Miskovic D, Allison AS et al (2016) Application of objective clinical human reliability analysis (OCHRA) in assessment of technical performance in laparoscopic rectal cancer surgery. Tech Coloproctol 20:361-367

2. Sabour S (2016) Application of objective clinical human reliability analysis (OCHRA) in assessment of technical performance in laparoscopic rectal cancer surgery: common mistakes and methodological issues. Tech Coloproctol. doi:10.1007/s10151-016-1540-5 